\title{
Highly Fluorescent Pyrene-Functional Polystyrene Copolymer Nanofibers for Enhanced Sensing Performance of TNT
}

\author{
Anitha Senthamizhan, ${ }^{\dagger}$ Asli Celebioglu, ${ }^{\dagger}$ Sumeyra Bayir, ${ }^{\ddagger}$ Mesut Gorur, ${ }^{*}$, Erdinc Doganci, ${ }^{\ddagger}, \|$ \\ Faruk Yilmaz, ${ }^{*}$, and Tamer Uyar, ${ }^{*}, \perp$ \\ ${ }^{\dagger}$ UNAM-National Nanotechnology Research Center, ${ }^{\perp}$ Institute of Materials Science \& Nanotechnology, Bilkent University, Ankara \\ 06800, Turkey \\ ${ }^{\ddagger}$ Department of Chemistry, Gebze Technical University, Kocaeli 41400, Turkey \\ ${ }^{\S}$ Department of Chemistry, Istanbul Medeniyet University, Istanbul 34700, Turkey \\ "Department of Science Education, Kocaeli University, Kocaeli 41380, Turkey
}

\section{Supporting Information}
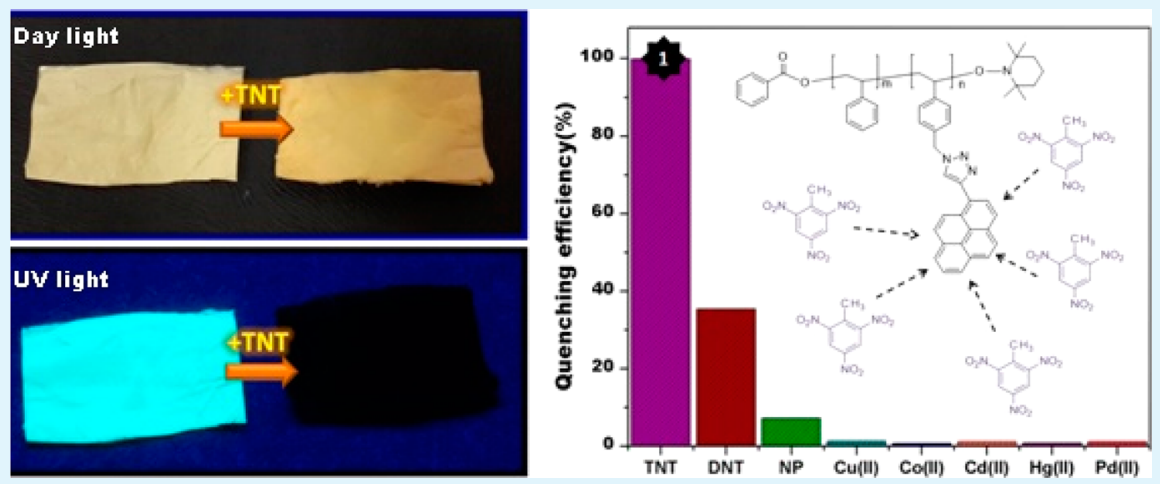

ABSTRACT: A pyrene-functional polystyrene copolymer was prepared via 1,3-dipolar cycloaddition reaction (Sharpless-type click recation) between azide-functional styrene copolymer and 1-ethynylpyrene. Subsequently, nanofibers of pyrene-functional polystyrene copolymer were obtained by using electrospinning technique. The nanofibers thus obtained, found to preserve their parent fluorescence nature, confirmed the avoidance of aggregation during fiber formation. The trace detection of trinitrotoluene (TNT) in water with a detection limit of $5 \mathrm{nM}$ was demonstrated, which is much lower than the maximum allowable limit set by the U.S. Environmental Protection Agency. Interestingly, the sensing performance was found to be selective toward TNT in water, even in the presence of higher concentrations of toxic metal pollutants such as $\mathrm{Cd}^{2+}, \mathrm{Co}^{2+}, \mathrm{Cu}^{2+}$, and $\mathrm{Hg}^{2+}$. The enhanced sensing performance was found to be due to the enlarged contact area and intrinsic nanoporous fiber morphology. Effortlessly, the visual colorimetric sensing performance can be seen by naked eye with a color change in a response time of few seconds. Furthermore, vapor-phase detection of TNT was studied, and the results are discussed herein. In terms of practical application, electrospun nanofibrous web of pyrene-functional polystyrene copolymer has various salient features including flexibility, reproducibility, and ease of use, and visual outputs increase their value and add to their advantage.

KEYWORDS: pyrene, colorimetric detection, TNT, nanofibers, toxic metals

\section{INTRODUCTION}

In the near future, several health and ecological risks have been posed by the increased use of explosives in the environment. Trinitrotoluene (TNT) is the most common explosive, which is also considered to be a hazardous waste by the U.S. Environmental Protection Agency (EPA), with a maximum permissible level in drinking water to be $2 \mathrm{ppb} .{ }^{1}$ The increased amount of TNT in water severely affects living organisms and causes several diseases like headache, anemia, and skin irritation, and the excess amount of TNT results in serious liver, eye, and neurological damage. This calls for an immediate need for the development of a rapid and reliable sensor for the detection of buried unexploded ordnance and for locating underwater mines. However, detection of trace amounts of TNT in aqueous environment faces significant challenges even today.

Until now, moderate efforts for the detection of TNT have been carried over using various kinds of analytical tools including mass spectrometry, electrochemical methods, surface enhanced Raman scattering methods, and fluorescence sensor. $^{2-8}$ Nevertheless, lack of portability, cost, time, sample preparation, and stability have restricted most of the devised

Received: February 25, 2015

Accepted: September 3, 2015

Published: September 3, 2015 
techniques for outdoor practical applications. Interestingly, owing to a number of advantages including high sensitivity, easy visualization, short response time, and low cost, more attention has been laid on colorimetric and fluorimetric-based detection approaches. $^{9-12}$ In addition, several fluorescent probes have also been designed for the enhanced detection of TNT.

In light on practicability, solid-state based sensors are more preferable than solution-based sensors. ${ }^{13-16}$ In general, fluorescent probes have been known for their ready attachment to the solid support for practical application. However, there have been issues concerning their stability due to leaching, reproducibility, and reduced lifetime. When compared to other fluorescent probes, polymer-based sensors have found a more attractive place than others owing to their easy processing attitude into larger area films through simpler techniques at lower cost with high repeatability rate. ${ }^{17,18}$

Pyrene functional macromolecules have been used widely as fluorescent probes $^{19-21}$ due to well-defined and excellent fluorescence properties of pyrene, such as relatively long fluorescence lifetime, forming excimers, and sensitivity to polarity of the environment. ${ }^{22-30}$ Pyrene-functional small molecular compounds, dendrimers, and polymers have been employed in chemical sensor applications based on fluorescence mechanism toward various anions, ${ }^{31-35}$ cations, ${ }^{36,37}$ biological entities, ${ }^{38}$ as well as nitroaromatic compounds. ${ }^{39,40}$ However, the number of papers on the fluorescence detection of nitroaromatics using electrospun pyrene-containing materials is very rare. Wand and co-workers reported a polystyrene fluorogenic nanofibrous sensing material from polystyrene/ pyrene blends for the detection of buried explosives. ${ }^{41}$ Guo and co-workers prepared polyvinylpyrrolidone/pyrene/3-aminopropyltriethoxysilane/reduced graphene oxide nanonets by electrospinning from their blends and reported that the nanonets gave quenching efficiency of $81 \%$ toward TNT vapor (10 ppb) within $540 \mathrm{~s}$ at room temperature. ${ }^{10}$ Hua et al. prepared electrospun polyacrylonitrile nanofibers coated with pyrene molecules for the detection of TNT based on fluorescence quenching mechanism. ${ }^{42}$

In one of our previous studies, ${ }^{43}$ we prepared $\alpha$-thiophene end-capped styrene copolymers containing pyrene side groups and investigated their fluorogenic responses in the presence of various anionic species at different concentrations. In this work, we studied the electrospinning of nanofibers from pyrenefunctional polystyrene copolymer for TNT detection in both aqueous and vapor-phase mediums. The main objective of this investigation is to study the feasibility of using functional polymeric nanofibrous membrane for the detection of TNT in wastewater as well as in vapor phase.

\section{EXPERIMENTAL DETAILS}

Materials. Styrene (Aldrich, 99.9\%) and 4-vinylbenzyl chloride (VBC, Aldrich, 90\%) were passed through a column of alumina to remove the inhibitor and were then stored under argon. Benzoyl peroxide (BPO, Aldrich, 98\%) was recrystallized from methanol and dried before use. 2,2,6,6-Tetramethyl-1-piperidinyloxy (TEMPO, Aldrich, 98\%), dimethylformamide (DMF, Merck, 99.8\%), sodium azide $\left(\mathrm{NaN}_{3}\right.$, Sigma-Aldrich, 99.5\%), $N, N, N^{\prime}, N^{\prime \prime}, N^{\prime \prime}$-pentamethyldiethylenetriamine (PMDETA, Aldrich, 99\%), copper(I) bromide ( $\mathrm{CuBr}$, Sigma-Aldrich, 98\%), 1-ethynylpyrene (Alfa Aesar, 96\%), dichloromethane (DCM, Merck, 99.8\%), and methanol (Merck, 99.9\%) were used without further purification.

Characterization. ${ }^{1} \mathrm{H}$ NMR $(500 \mathrm{MHz})$ spectra were obtained with a Varian Unity INOVA spectrometer at $25^{\circ} \mathrm{C}$ in $\mathrm{CDCl}_{3}$ solutions relative to the nondeuterated solvent traces as the internal reference.
FT-IR spectra were collected on a PerkinElmer Spectrum Two spectrometer equipped with UATR accessory. The molecular weights of the synthesized polymers were determined by gel permeation chromatography (GPC) using an Agilent 1260 Infinity GPC/SEC instrument consisting of a pump, a refractive index detector, and two Agilent PLgel columns (Mixed-C, $5 \mu \mathrm{m}, 7.5 \times 300 \mathrm{~mm}$ ) at $40{ }^{\circ} \mathrm{C}$, which was calibrated with linear polystyrene standards. Tetrahydrofuran was used as the eluent at a flow rate of $1 \mathrm{~mL} / \mathrm{min}$. The glass transition temperatures $\left(T_{\mathrm{g}}\right)$ of the synthesized polymers were measured with DSC 8500 (PerkinElmer) instrument under a nitrogen flow of $10 \mathrm{~mL} / \mathrm{min}$. The samples were first heated from 25 to $160{ }^{\circ} \mathrm{C}$, then cooled to $25{ }^{\circ} \mathrm{C}$, and finally heated to $160{ }^{\circ} \mathrm{C}$ at a scan rate of 10 ${ }^{\circ} \mathrm{C} \mathrm{min}^{-1}$. Thermal stability measurements of the polymers were performed with a TGA/SDTA 851 (Mettler Toledo) thermogravimetric analyzer from room temperature to $700{ }^{\circ} \mathrm{C}$ at a heating rate of $10{ }^{\circ} \mathrm{C} \mathrm{min}^{-1}$ under nitrogen atmosphere. The morphology of the electrospun nanofibers were analyzed by using scanning electron microscope (Quanta $200 \mathrm{FEG}$ ). The fluorescence emission characteristics were measured by time-resolved fluorescence spectrophotometer (FL-1057 TCSPC). The thickness of the nanofibers and films were measured by using digital micrometer.

Synthesis of Chloride-Functional Styrene Polymer (P1). Styrene (6.97 g, $66.92 \mathrm{mmol})$, 4-vinylbenzyl chloride (1.13 g, 7.43 $\mathrm{mmol})$, and TEMPO $(0.009 \mathrm{~g}, 0.06 \mathrm{mmol})$ were put into a onenecked round-bottom flask and stirred for 10 min under gentle argon purge. BPO $(0.010 \mathrm{~g}, 0.031 \mathrm{mmol})$ was added, and the flask was tightly closed and then immersed in a thermostated oil bath at $120^{\circ} \mathrm{C}$; the mixture continued stirring for $48 \mathrm{~h}$. After the reaction mixture was cooled to room temperature, the crude polymerization product was dissolved in DCM $(15 \mathrm{~mL})$ and purified by precipitating in cold methanol. P1 was recovered by vacuum filtration through a sintered glass filter (G4) and dried under reduced pressure at $35^{\circ} \mathrm{C}$ for $48 \mathrm{~h}$.

Yield. $5.75 \mathrm{~g}(69.5 \%) . M_{\mathrm{n}, \mathrm{GPC}}: 90500 \mathrm{~g} / \mathrm{mol} ; M_{\mathrm{w}} / M_{\mathrm{n}}: 1.23$. FT-IR $\left(\mathrm{cm}^{-1}\right)$ : 3027-3063 (CH stretching, aromatic); 2848-2921 $(\mathrm{CH}$ stretching, aliphatic). ${ }^{1} \mathrm{H}$ NMR $\left(500 \mathrm{MHz}, \mathrm{CDCl}_{3}, \delta\right.$, ppm): 6.46$7.09\left(\mathrm{C}_{6} \mathrm{H}_{4}\right.$ and $\left.\mathrm{C}_{6} \mathrm{H}_{5}\right) ; 4.51\left(\mathrm{C}_{6} \mathrm{H}_{4} \mathrm{CH}_{2} \mathrm{Cl}\right) ; 1.42-2.04$ (polymer backbone).

Synthesis of Azide-Functional Styrene Polymer (P2). P1 (4.5 $\mathrm{g}$, contains $4.38 \mathrm{mmol} \mathrm{Cl})$ was dissolved in DMF $(50 \mathrm{~mL})$ under argon atmosphere. $\mathrm{NaN}_{3}(2.63 \mathrm{~g}, 40.46 \mathrm{~mol})$ was then added, and the reaction mixture was degassed with a slow stream of argon for $10 \mathrm{~min}$ and placed in an oil bath thermostated at $80^{\circ} \mathrm{C}$ with stirring for $48 \mathrm{~h}$. After the mixture was allowed to cool to ambient temperature, it was transferred to a separatory flask with DCM $(100 \mathrm{~mL})$ and then washed with water $(2 \times 100 \mathrm{~mL})$. The organic phases were then combined, dried over $\mathrm{MgSO}_{4}$, concentrated to $\sim 10 \mathrm{~mL}$ with rotary evaporation, and precipitated into cold methanol. P2 was recovered by vacuum filtration through a sintered glass filter (G4) and dried under reduced pressure at $35{ }^{\circ} \mathrm{C}$ for $48 \mathrm{~h}$.

Yield. $3.62 \mathrm{~g}(80.0 \%) . M_{\mathrm{n}, \mathrm{GPC}}: 90800 \mathrm{~g} / \mathrm{mol} ; M_{\mathrm{w}} / M_{\mathrm{n}}: 1.25$. FT-IR $\left(\mathrm{cm}^{-1}\right)$ : 3027-3063 (CH stretching, aromatic); 2848-2921 (CH stretching, aliphatic); $2095\left(\mathrm{~N}_{3}\right) .{ }^{1} \mathrm{H}$ NMR $\left(500 \mathrm{MHz}, \mathrm{CDCl}_{3}, \delta\right.$, ppm): 6.46-7.09 $\left(\mathrm{C}_{6} \mathrm{H}_{4}\right.$ and $\left.\mathrm{C}_{6} \mathrm{H}_{5}\right) ; 4.22\left(\mathrm{C}_{6} \mathrm{H}_{4} \mathrm{CH}_{2} \mathrm{~N}_{3}\right) ; 1.42-2.04$ (polymer backbone).

Synthesis of Pyrene-Functional Styrene Polymer (P3). P2 (4.0 g, contains $3.87 \mathrm{mmol} \mathrm{N}_{3}$ ) and 1-ethynylpyrene (1.05 g, 4.64 $\mathrm{mmol})$ were dissolved in DMF $(50 \mathrm{~mL})$ under an argon atmosphere. PMDETA (2.01 g, $11.60 \mathrm{mmol}$ ) was added, and the reaction mixture was degassed by gently purging with oxygen-free argon for $5 \mathrm{~min}$. Subsequently, $\mathrm{CuBr}(1.66 \mathrm{~g}, 11.60 \mathrm{mmol})$ was added to the solution, and the solution was degassed again. After the reaction mixture was stirred at room temperature for $48 \mathrm{~h}$, it was transferred to an extraction funnel, diluted with DCM $(100 \mathrm{~mL})$, and washed successively with and water $(2 \times 100 \mathrm{~mL})$. The collected organic phases were dried over $\mathrm{MgSO}_{4}$, concentrated to $\sim 10 \mathrm{~mL}$ by a rotary evaporator, and $\mathbf{P} 3$ was isolated by precipitation from cold methanol. The obtained polymer was collected by vacuum filtration through a sintered glass filter (G4) and dried under reduced pressure at $35{ }^{\circ} \mathrm{C}$ for $48 \mathrm{~h}$.

Yield. $3.89 \mathrm{~g}$ (79.9\%). $M_{\mathrm{n}, \mathrm{GPC}}: 91300 \mathrm{~g} / \mathrm{mol} ; M_{\mathrm{w}} / M_{\mathrm{n}}: 1.28$. FT-IR $\left(\mathrm{cm}^{-1}\right)$ : 3027-3063 (CH stretching, aromatic); 2848-2921 (CH 
Scheme 1. General Reaction Scheme for the Synthesis of Styrene Copolymer Containing Pyrene Side Groups<smiles>C=Cc1ccc(CCl)cc1</smiles><smiles>CC(C)(OC(=O)c1ccccc1)OC(C)(C)C(c1ccccc1)C(C)(C)C(C)(C)C(C)(C)ON1C(C)(C)CCCC1(C)C</smiles>

P1

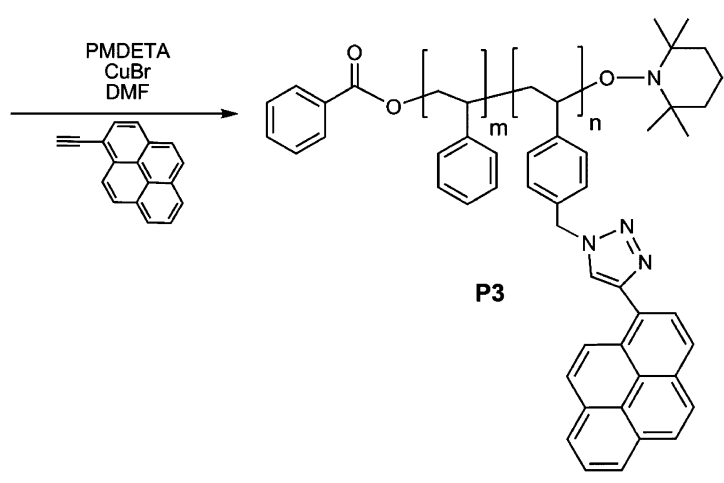

stretching, aliphatic). ${ }^{1} \mathrm{H}$ NMR $\left(500 \mathrm{MHz}, \mathrm{CDCl}_{3}, \delta, \mathrm{ppm}\right): 7.68-$ 8.68 ( $\mathrm{CH}$, pyrene); 6.46-7.09 $\left(\mathrm{C}_{6} \mathrm{H}_{4}\right.$ and $\left.\mathrm{C}_{6} \mathrm{H}_{5}\right) ; 5.22\left(\mathrm{C}_{6} \mathrm{H}_{4} \mathrm{CH}_{2}-\right.$ $\mathrm{C}_{2} \mathrm{HN}_{3}$ ); $1.42-2.04$ (polymer backbone).

Electrospinning of the Fluorescence Nanofibers. The electrospinning solution of pyrene functional polystyrene copolymer (P3) was obtained by dissolving in DMF/DCM (7/3 (v/v)) solvent mixture at $12 \%(\mathrm{w} / \mathrm{v}$, with respect to solvent) concentration. The homogeneous clear solution was placed in a $1 \mathrm{~mL}$ syringe fitted with metallic needles of $0.4 \mathrm{~mm}$ inner diameter. This was followed by the horizontal fixing of the syringe on the syringe pump (model SP 101IZ, WPI). The polymer solution was pumped with a feed rate of $0.5 \mathrm{~mL} / \mathrm{h}$ during electrospinning. By using a high voltage supply, the applied voltage to the metal needle tip (Spellman, SL Series) was $15 \mathrm{kV}$ and the tip-to-collector distance was set at $15 \mathrm{~cm}$ for electrospinning of the prepared solution. Collectively, electrospun fluorescence $\mathbf{P} 3$ nanofibers were deposited on the aluminum foil covering the plate-type collector. The electrospinning process was performed at $20{ }^{\circ} \mathrm{C}$ and $20 \%$ relative humidity in an enclosed Plexiglas box. For comparison studies, the solution used for electrospinning was drop-casted on the glass Petri dish and then allowed for drying up to 3 days in a vacuum oven at 70 ${ }^{\circ} \mathrm{C}$. The dried film was peeled from the dish as a free-standing film and then used for further experiments.

Visual Colorimetric Detection of Analytes in Water. At a concentration of $5 \mathrm{mM}$, a stock solution of TNT is prepared by dissolving TNT in water/acetonitrile $\left(\mathrm{H}_{2} \mathrm{O} / \mathrm{ACN}, 1: 1\right)$ mixture. This is followed by diluting different concentrations of TNT from the stock solution. Visual colorimetric detection is done by cutting the fluorescent fibrous membrane into small pieces and then dipping the same for $5 \mathrm{~min}$ in different concentrations of TNT solution. In few seconds, color changes were noticed by the naked eye. After evaporation of the solvent, a photograph was taken under UV light $\left(\lambda_{\text {ext }}-254 \mathrm{~nm}\right)$ and normal light conditions. The same procedure was followed for DNT and other metal ions.

Vapor-Phase Sensing of Trinitrotoluene. In brief, $1.5 \mathrm{~g}$ of TNT is taken into a quartz cuvette and then covered with a small piece of cotton to avoid direct contact with fluorescent probes, that is, nanofibrous membrane. The setup is allowed for saturation for $48 \mathrm{~h}$ and then placed in a time-resolved fluorescence spectrophotometer. A small piece of the membrane is placed into the quartz cuvette, and immediate changes in fluorescence are recorded at the desired time period. The excitation wavelength was fixed at $340 \mathrm{~nm}$.

\section{RESULTS AND DISCUSSION}

Synthesis and Characterization of Pyrene Functional Polystyrene Copolymer. Pyrene-functional polystyrene copolymer (P3) was prepared in a three-step synthetic procedure (Scheme 1). In the first step, chloride-functional styrene copolymer (P1) was synthesized via nitroxy-mediated stable free radical polymerization (NMP) of styrene and 4vinylbenzyl chloride as monomers using $\mathrm{BPO}$ as the radical initiator and TEMPO as the coradical. Then, chloride side groups were converted into azide by reacting P1 with sodium azide in DMF, yielding azide-functional styrene copolymer (P2). In the last step, pyrene-functional styrene copolymer (P3) was obtained quantitatively through 1,3-dipolar cycloaddition reaction (click reaction) between azide-functional groups of P2 and 1-ethynylpyrene.

The chemical structures of the synthesized copolymers (P1P3) were confirmed via FT-IR and ${ }^{1} \mathrm{H}$ NMR spectral analysis. In their FT-IR spectra given in Figure S1, aromatic and aliphatic stretching bands are observed around 3027-3063 and $2848-2921 \mathrm{~cm}^{-1}$, respectively. In the FT-IR spectrum of P2, the signal observed at $2095 \mathrm{~cm}^{-1}$ clearly indicates the presence of azide functional groups in the chemical structure of $\mathbf{P 2}$. Upon click reaction between $\mathbf{P 2}$ and 1-ethynylpyrene, the complete disappearence of azide signal, which was observed in the FT-IR spectrum of the precursor, proves that pyrene side groups were attached quantitatively, yielding P3. As for the ${ }^{1} \mathrm{H}$ NMR spectra of the copolymers (Figure S2), the backbone 

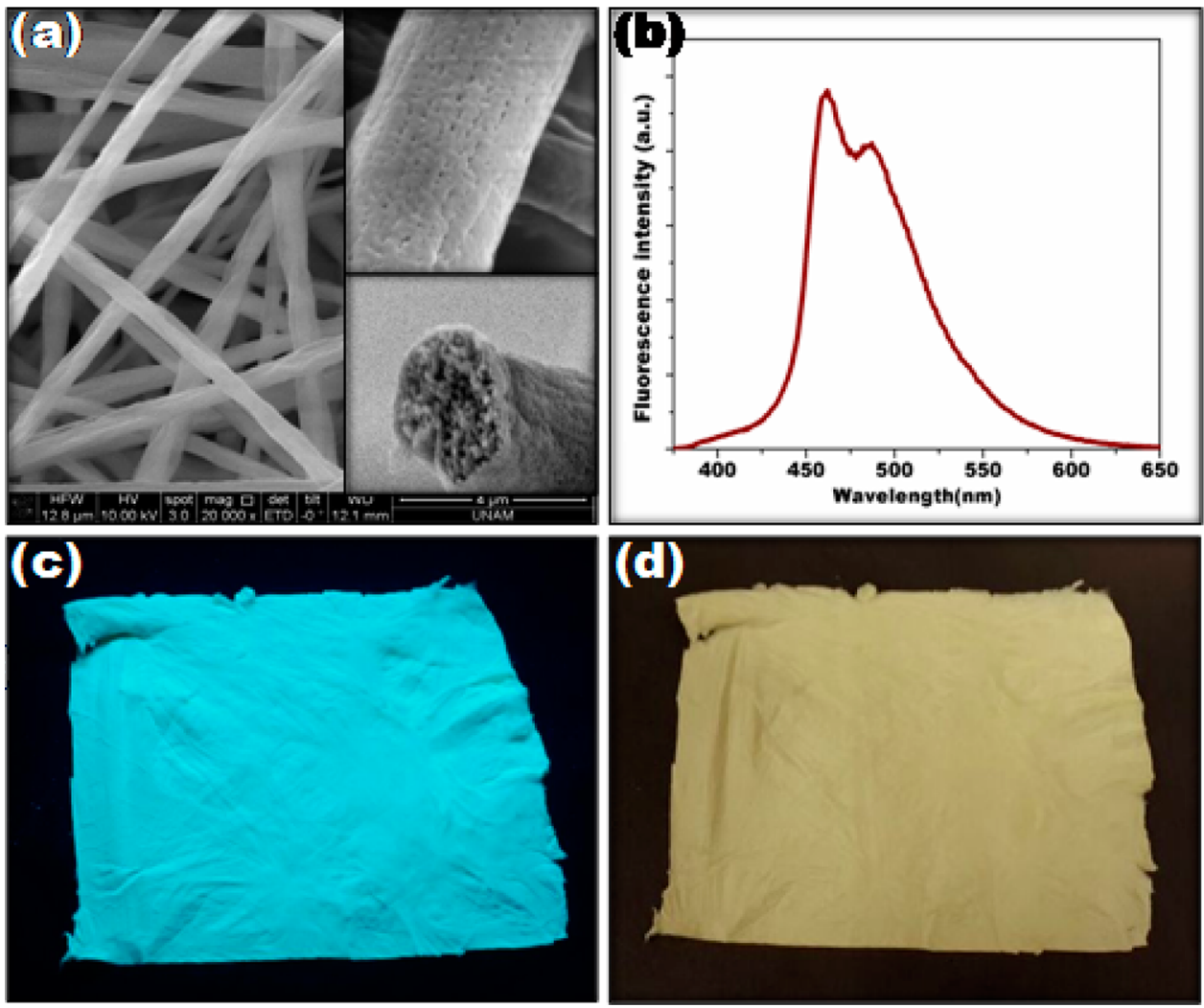

Figure 1. (a) SEM image of the pyrene functional polystyrene copolymer nanofibers. (inset) Porous nature and cross-sectional view of the nanofiber. (b) Fluorescence emission spectra $\left(\lambda_{\text {ext }}-340 \mathrm{~nm}\right)$. (c) Photograph of FNFM under UV light $\left(\lambda_{\text {ext }}-254 \mathrm{~nm}\right)$ and (d) daylight.

protons $\left(\mathrm{H}_{\mathrm{a}}\right)$ gave signals between 1.42 and $2.04 \mathrm{ppm}$, while aromatic $\mathrm{CH}$ protons $\left(\mathrm{H}_{\mathrm{c}}\right)$ in styrene and vinylbenzyl repeating units resonanced between 6.46 and $7.09 \mathrm{ppm}$. In the ${ }^{1} \mathrm{H}$ NMR spectrum of $\mathbf{P 1}$, the signal of the methylene protons $\left(\mathrm{H}_{\mathrm{b}}\right)$ next to the benzene ring was observed at $4.51 \mathrm{ppm}$, and it was shifted to higher magnetic field $(4.22 \mathrm{ppm})$ in the ${ }^{1} \mathrm{H}$ NMR spectrum of P2 upon azidification. After click reaction of P2 with 1-ethynylpyrene, the chemical environment of these protons $\left(\mathrm{H}_{\mathrm{b}}\right)$ changed, and they gave resonances at 5.52 ppm. The clear shift of $\mathrm{H}_{b}$ protons on azidification and click reactions, explicitly proves the success of the reactions. Besides, pyrene proton signals were observed between 7.68 and 8.68 ppm in the ${ }^{1} \mathrm{H}$ NMR spectrum of $\mathbf{P 3}$.

Average molecular weights of the styrene copolymers (P1P3) were estimated by conventional gel permeation chromatography, and the obtained data were presented in Table S1. The polydispersity values ranged between 1.23 and 1.28 , whereas the average molecular weights from GPC experiments are estimated values based on unfunctional polystyrene calibration standards. The hydrodynamic volumes of the functional styrene copolymers (P1-P3) were different, to some extent, from the calibration standards. Thus, average molecular weight values from GPC experiments are accepted to be less reliable than those obtained from ${ }^{1} \mathrm{H}$ NMR calculations. In the ${ }^{1} \mathrm{H}$ NMR spectra of the styrene copolymers (P1-P3), the signals of BPO and TEMPO residues were overlapped by those of the repeating units or were very weak due to dilution of terminal units in long polymer chains. Fortunately, the ratio between monomeric residues $(m / n$, see Scheme 1$)$ in the styrene copolymers could be calculated from the integral ratios of aromatic phenyl proton signals $\left(\mathrm{C}_{6} \mathrm{H}_{5}\right.$ plus $\left.\mathrm{C}_{6} \mathrm{H}_{4}-\mathrm{CH}_{2}-\right)$ and methylene protons next to the benzene ring $\left(\mathrm{C}_{6} \mathrm{H}_{4}-\mathrm{CH}_{2}\right)$ and were summarized in Table S1. Therefore, the molar mass of the fragments containing single functional unit $\left(-\mathrm{Cl}\right.$ or $\left.-\mathrm{N}_{3}\right)$ were calculated using the equation $(m / n) \times \mathrm{MW}$ of styrene $+\mathrm{MW}$ of chloride or azide functional repeating units.

Differential scanning calorimetry (DSC) technique was used to determine thermal transitions, such as $T_{g}$, of the synthesized styrene copolymers with different side-functional groups. Figure S3 shows the DSC curves of the styrene copolymers in the second heating run, and the related data were given in Table S2. $T_{\mathrm{g}}$ values of the chloride functional styrene copolymer (P1) was measured as $\sim 104{ }^{\circ} \mathrm{C}$. Upon azidification, $T_{\mathrm{g}}$ of the styrene copolymer (P2) decreased slightly to $\sim 99{ }^{\circ} \mathrm{C}$. However, attachment of pyrene side groups considerably increased $T_{\mathrm{g}}$ $\left(\sim 126^{\circ} \mathrm{C}\right)$ of the resultant fluorescent styrene copolymer (P3), due to rigid structure and $\pi$-stacking of pyrene side-units. As for the thermogravimetric properties of the copolymers, their maximum decomposition temperatures $\left(T_{\mathrm{Max}}\right)$ of the styrene copolymers were very close to each other as seen from Figure S4, indicating that their thermal stabilities do not differ considerably. Besides, the percent char yield of the pyrenefunctional styrene copolymer was remarkably greater than the others.

Trinitrotoluene Sensing Applications of Electrospun Pyrene Functional Polystyrene Copolymer. The schematic representation of electrospinning of nanofibers from pyrenefunctional polystyrene copolymer (P3) is shown in Figure S5. The SEM image of electrospun pyrene-functional styrene copolymer (P3) described as fluorescent nanofiber (FNF) depicts a uniform defect-free porous structure with average fiber diameter of $400 \pm 140 \mathrm{~nm}$ as illustrated in Figure 1a. The presence of pores is clearly seen on surface of the nanofibers with an average pore size of $30-40 \mathrm{~nm}$. And also cross- 
sectional view of the fiber confirms the occurrence of porous structure inside the fiber. The fluorescent polymer demonstrated excimer emissions mainly due to increased local concentration of pyrene moieties on the polymer backbone as shown in Figure $1 \mathrm{~b}$ and Figure S6.

As compared with photoluminescent spectra of fluorescent polymer in solution phase (Figure S6), the fibrous film did not shift much to higher wavelength as observed by others. ${ }^{4-46}$ This result confirmed that the aggregation enhanced excimer emission is limited. The nanofibers thus obtained, found to preserve their parent fluorescence nature, confirmed the avoidance of extended aggregation during fiber formation. Further, the fluorescent nanofibrous membrane (FNFM) exhibited bright bluish-green emission, visually observed under UV illumination $\left(\lambda_{\text {ext }}-254 \mathrm{~nm}\right)$ as shown in Figure 1c, and their depiction under normal light conditions is displayed in Figure 1d.

Importantly, even after treatment with water for a prolonged period of time, the FNFM does not lose its fluorescence nature. Furthermore, the sensing performance of FNFM, was taken into testing by dipping the membrane in different concentrations of aqueous TNT solutions (in $\mathrm{H}_{2} \mathrm{O} / \mathrm{ACN}(1: 1)$ ) for 10 min, presented in Figure 2. The corresponding fluorescence spectra before and after the treatment of TNT, with a concentration ranging from $5 \mathrm{mM}$ to $5 \mathrm{nM}$, clearly shows changes in fluorescence nature with respect to their

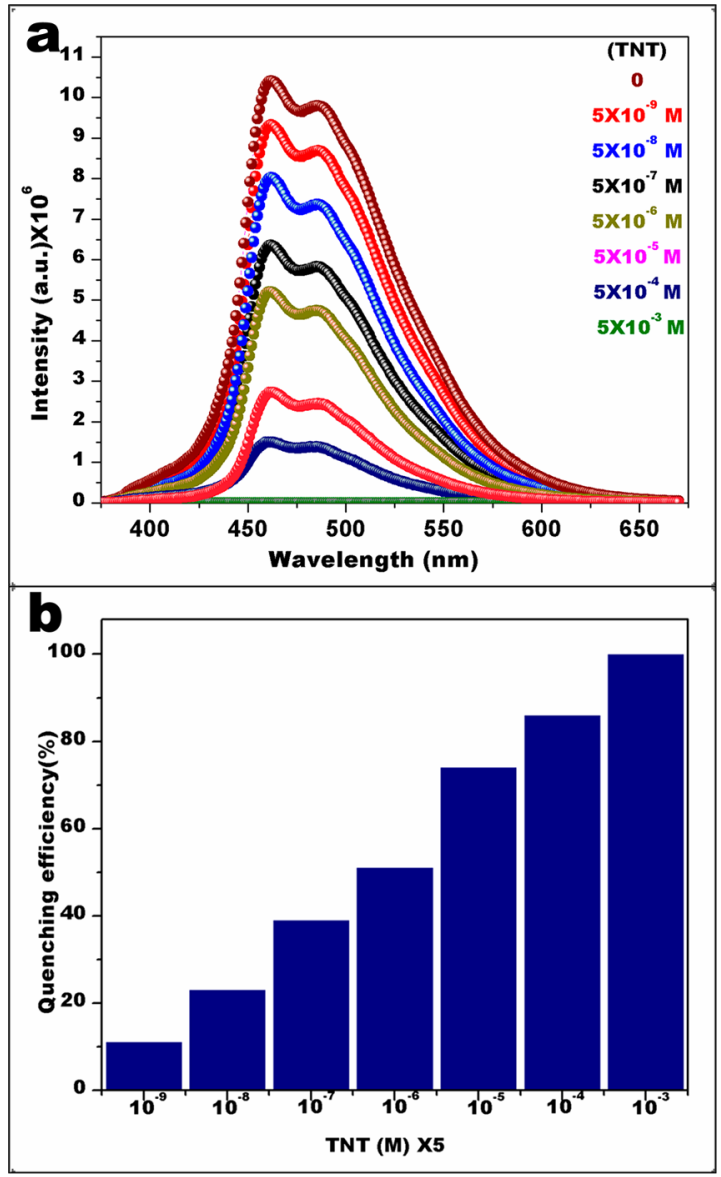

Figure 2. (a) Fluorescence emission spectra of FNFM upon exposure to various concentrations of TNT and their (b) quenching efficiency. The increase in the concentration of TNT is seen to enhance the quenching efficiency. concentration as illustrated in Figure 2a. The quenching efficiency $\left(\mathrm{QE}=\left(I_{0}-I\right) / I_{0} \times 100\right.$, where $I$ and $I_{0}$ are the fluorescence intensity in the presence and absence of TNT, respectively) versus TNT concentration is shown in Figure 2b, where a clear representation of the gradual increase in the concentration of TNT is seen to enhance the quenching efficiency. Also, it is found that the limit of detection reached 5 $\mathrm{nM}$, lower than the maximum permissible limit of TNT (10 $\mathrm{nM}$ ) in drinking water set by the EPA.

Further, visual colorimetric detection was performed for different concentrations of TNT from $5 \mathrm{mM}$ to $5 \mathrm{nM}$. Apparently, the visual colorimetric sensing performance of FNFM is easily identifiable under UV light $\left(\lambda_{\text {ext }}-254 \mathrm{~nm}\right)$ illumination by naked eye, since the color of the membrane changes from bright bluish-green to blue at selected concentrations. Further, maintaining the same and normal light conditions, their photographs were taken, presented in Figure 3. It is apparent that color change has happened from light sandal to dark sandal at higher concentration under normal light conditions. Notably, color change was identified within the first few minutes indicating their rapid response character, and the limit of visual detection was found to be $5 \times$ $10^{-5} \mathrm{M}$. Initially, this step was initiated to check color change in the presence of the solvent that is used for dissolving TNT. As anticipated, no color change was noticed. After subsequent treatment with TNT, morphology of the fibrous membrane was studied, and the results confirmed that the nanofibrous morphology of the membrane did not get destroyed even at higher concentrations as shown in Figure S7.

Further, to indicate the importance of selective response for real-life application of a sensor in aqueous phase, we investigated the interference from other nitro aromatic compounds and commonly found toxic metals in water. Remarkably, the results highlighted that the presence of 2,4dinitrotoluene (DNT) at a concentration of $1 \mathrm{mM}$ decreases the fluorescence intensity considerably, and 4-nitrophenol (NP) also slightly decreases the fluorescence intensity. Interestingly, at this concentration, TNT is found to effectively quench the entire fluorescence of the FNFM indicating their enhanced sensing response. Apart from this, no significant change in fluorescence emission was noticed in the presence of toxic metal ions visually as well as spectroscopically, clearly demonstrated in Figure 4. The observed selective sensing performance might be attributed to the screening effect of hydrophobic polystyrene fibers, hindering the interaction of metal ions with pyrene units. Therefore, the exceptional characteristics along with their selective visual colorimetric sensing performance have an excellent potential to be in use as a portable sensor for the effective detection of TNT in water for environmental applications.

Upon exposure to TNT vapor at room temperature, the time-dependent fluorescence quenching of FNFM was evaluated and presented in Figure 5. As shown in the spectra, it is evident that the quenching is started within $30 \mathrm{~s}$ of TNT exposure, and its persistent decrease with increasing time is displayed in Figure 5a. The corresponding relative fluorescence intensity $I_{0} / I$ versus TNT concentration is shown in Figure $5 b$. The inset in Figure $5 \mathrm{~b}$ confirms the linearity of the sensing response over time period of $600 \mathrm{~min}$. When viewing by naked eye under the exposure of UV light, significant color change was noticed within $30 \mathrm{~min}$ of exposure as illustrated in Figure 5 inset, and the complete fluorescence quenching was observed for $48 \mathrm{~h}$ as shown in Figure S8. 

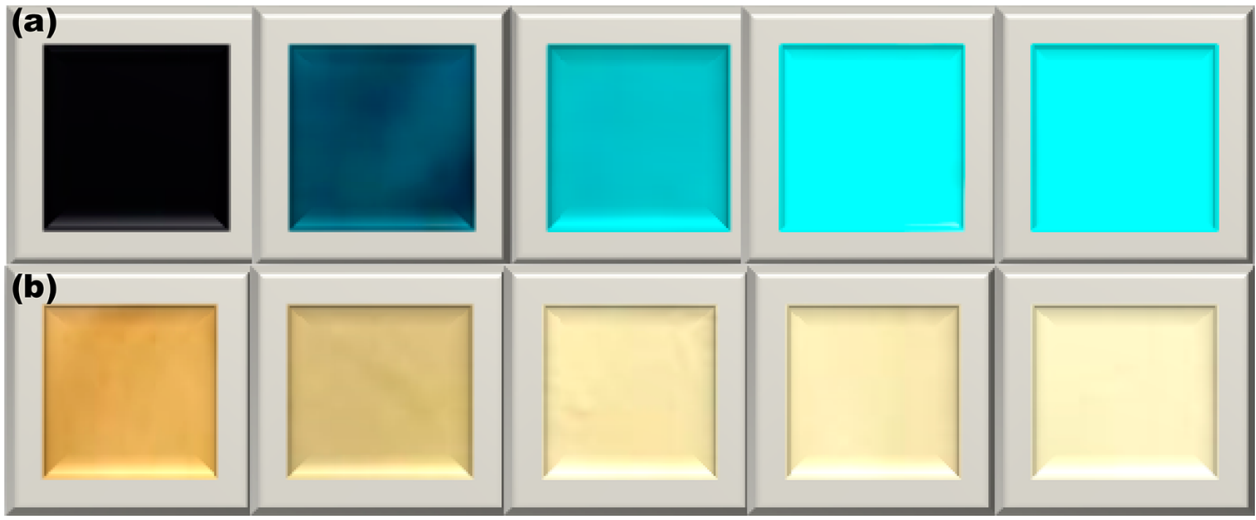

Figure 3. Visual colorimetric detection of TNT. Photograph of the fluorescence quenching of FNFM treated with different concentrations of TNT in aqueous phase when viewed under $\operatorname{UV}\left(\lambda_{\text {ext }}-254 \mathrm{~nm}\right)(\mathrm{a})$ and daylight $(\mathrm{b})$. The tested TNT concentrations are $5 \times 10^{-3}, 5 \times 10^{-4}, 5 \times 10^{-5}, 5 \times$ $10^{-6}$, and $5 \times 10^{-7} \mathrm{M}$ from left to right. The color of the membrane changes from bright bluish-green to blue with increasing concentration.
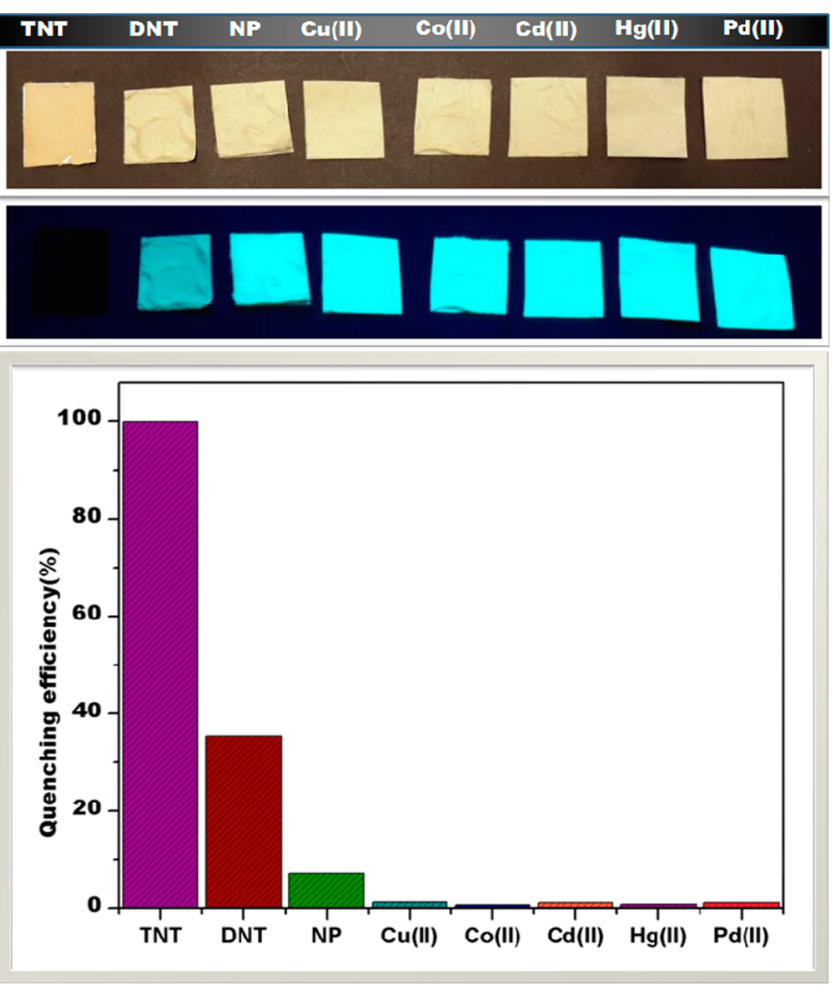

Figure 4. Selective sensing performance of FNFM upon exposure to other nitro aromatic compounds and toxic metal ions. The concentrations of DNT, NP, and TNT are fixed at $1 \mathrm{mM}$, and all other metal ions are fixed at $20 \mathrm{ppm}$. The presence of DNT decreases the fluorescence intensity considerably, and NP also slightly decreases the fluorescence intensity. Interestingly, at this concentration, TNT is found to effectively quench the entire fluorescence of the FNFM, and all other metal ions have no effect.

When compared with the fluorescence spectra, fluorescence intensity completely disappeared without affecting their nanofiber morphology, further confirmed by SEM analysis as shown in Figure S9. In addition, we are interested to know the advantage of nanofibers in sensing performance. Therefore, we selected solution casting thin film as a reference. The thin film is brittle in nature, and it can be easily broken as shown in Figure S10. The tested solution cast thin film and nanofibrous membrane were both in approximately equal thickness and size (thickness of $\sim 0.18 \mathrm{~mm}$ and size of $0.5 \times 1.5 \mathrm{~cm}$ ), but the
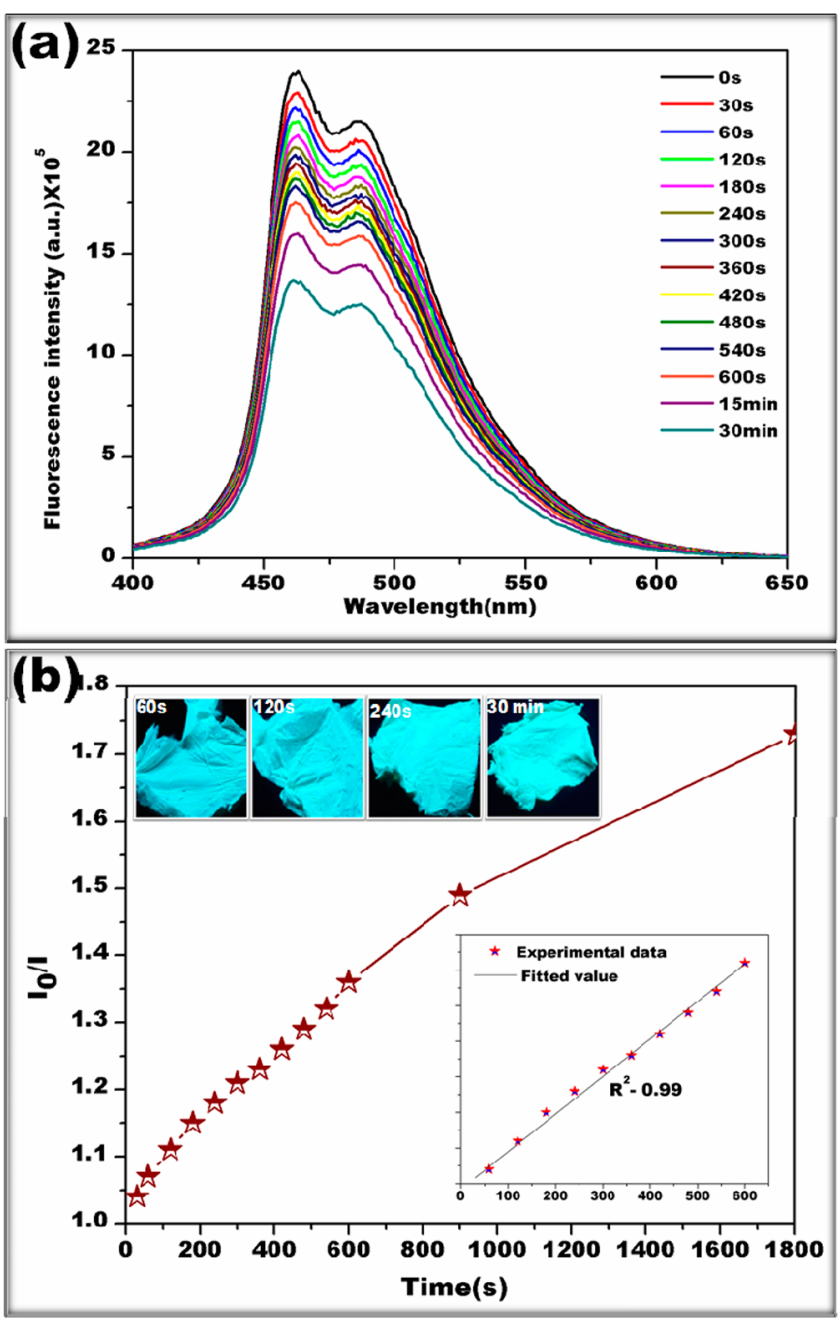

Figure 5. (a) Time-dependent fluorescent spectra of FNFM upon exposure to saturated TNT vapor with different time periods and their (b) relative fluorescence intensity. The significant color change was noticed within $30 \mathrm{~min}$ of exposure (inset).

weight of thin film $(50 \mathrm{mg})$ was 25 times higher than the nanofibrous membrane ( $2 \mathrm{mg}$ ).

The compared quenching efficiency toward TNT with a different time interval is given in Figure 6. The observed result confirms the quenching efficiency of nanofibrous membrane is 
3 times higher than the thin film even when much less material was used for the TNT detection.

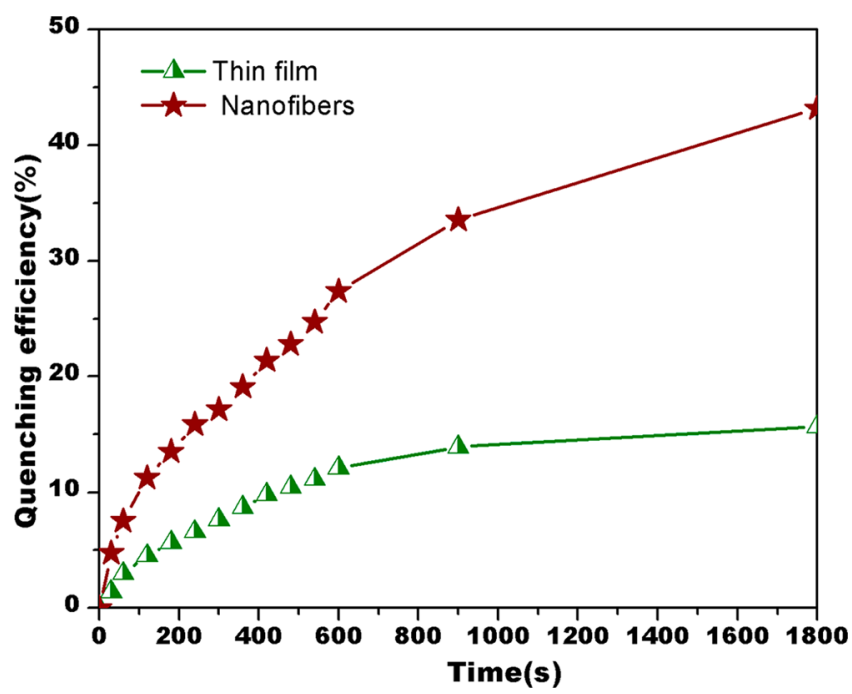

Figure 6. Compared time-dependent fluorescence quenching of the nanofibers and thin film.

Finally, the outstanding sensing performance in aqueous phase and the comparatively higher quenching efficiency in vapor phase might be attributed to the higher surface area of the nanofibers, resulting in enhanced interaction between TNT and its analytes. ${ }^{18,41,47-52}$ Reportedly, the presence of a conjugated planar structure of pyrene and a large delocalized $\pi$-system makes them more selective toward TNT. During TNT interaction with pyrene, because of similarities in their orbital energy levels, they could easily bind with one another via $\pi-\pi$ interaction, and the corresponding electrons in the pyrene are supposed to get transferred to the lowest unoccupied molecular orbital of the TNT. ${ }^{53,37}$ The resulting photoinduced electron transfer quenches the fluorescence of FNFM, which is found to be concentration-dependent. In addition, triazole bridge also plays a significant role in enhancing the sensing performance of TNT. The schematic representation of the sensing mechanism is illustrated in Figure S11. To confirm this, XPS spectra were taken for analyzing the interaction between triazole and TNT, and they are shown in Figure S12. The appearance of new peak at $\sim 406 \mathrm{eV}$ is assigned to the nitro group from TNT. In addition, upon increasing the concentration of TNT, there is a gradual shift in the spectra, which confirms the interaction between triazole proton and nitro group in $\mathrm{TNT}^{54-56}$

\section{CONCLUSIONS}

To summarize, successful demonstration of electrospun pyrenefunctional polystyrene copolymer nanofibrous membrane has been done for efficient trace detection of TNT in aqueous solution as well as vapor phase. The lowest detection limit has been found to be $5 \mathrm{nM}$, much lower than the maximum allowable limit set by the EPA. In the presence of other nitroaromatic compounds and competent metal ions, it has been analyzed that the sensing performance is more selective toward TNT in water. Within few seconds, a drastic color change has been noticed, easily identifiable by the naked eye. The underlying mechanism outlining the enhanced sensing performance has been studied to be the uniform one- dimensional morphology along with its intrinsic nanoporous structure. We have presented a promising area of research for sensor application highlighting their advantages of simplicity, specificity, high sensitivity, selectivity, and an economy with lower detection. In future, we expect to involve in optimizing the obtained fibrous system to improve the visual colorimetric sensing performance in vapor phase.

\section{ASSOCIATED CONTENT}

\section{Supporting Information}

The Supporting Information is available free of charge on the ACS Publications website at DOI: 10.1021/acsami.5b07184.

Styrene copolymers with various side-functional groups, thermal properties of styrene copolymers, FTIR, NMR, DSC, TGA, SEM, and XPS measurements, schematic of nanofiber formation, emission spectra, schematic of TNT sensing mechanism, and photographs. (PDF)

\section{AUTHOR INFORMATION}

\section{Corresponding Authors}

*E-mail: uyar@unam.bilkent.edu.tr, tameruyar@gmail.com. (T.U.)

*E-mail: fyilmaz@gtu.edu.tr. (F.Y.)

*E-mail: mesut.gorur@medeniyet.edu.tr. (M.G.)

\section{Author Contributions}

The manuscript was written through contributions of all authors. All authors have given approval to the final version of the manuscript.

\section{Notes}

The authors declare no competing financial interest.

\section{ACKNOWLEDGMENTS}

A.S. thanks the Scientific \& Technological Research Council of Turkey (TUBITAK; TUBITAK-BIDEB 2216, Research Fellowship Programme for Foreign Citizens) for postdoctoral fellowship. A.C. acknowledges TUBITAK (Project No. 113Y348) for postdoctoral funding. M.G., F.Y., and T.U. acknowledge TUBITAK (Project No: 113Z577) for financial support. T.U. also acknowledges partial support of Turkish Academy of Sciences-Outstanding Young Scientists Award Program (TUBA-GEBIP).

\section{REFERENCES}

(1) Agency for Toxic Substances and Disease Registry. Toxicological Profile for 2,4,6-Trinitrotoluene; U.S. Department of Health and Human Services, 1995.

(2) Lee, Y. H.; Liu, H.; Lee, J. Y.; Kim, S. H.; Kim, S. K.; Sessler, J. L.; Kim, Y.; Kim, J. S. Dipyrenylcalix[4]arene-A Fluorescence-Based Chemosensor for Trinitroaromatic Explosives. Chem. - Eur. J. 2010, 16, 5895-5901.

(3) Salinas, Y.; Agostini, A.; Perez-Esteve, E.; Martinez-Manez, R.; Sancenon, F.; Dolores Marcos, M.; Soto, J.; Costero, A. M.; Gil, S.; Parra, M.; Amoros, P. Fluorogenic Detection of Tetryl and TNT Explosives Using Nanoscopic-Capped Mesoporous Hybrid Materials. J. Mater. Chem. A 2013, 1, 3561-3564.

(4) Shaligram, S.; Wadgaonkar, P. P.; Kharul, U. K. Fluorescent Polymeric Ionic Liquids for the Detection of Nitroaromatic Explosives. J. Mater. Chem. A 2014, 2, 13983-13989.

(5) Zhang, L.; Li, C.; Liu, A.; Shi, G. Electrosynthesis of Graphene Oxide/Polypyrene Composite Films and their Applications for Sensing Organic Vapors. J. Mater. Chem. 2012, 22, 8438-8443. 
(6) Wang, X.; Drew, C.; Lee, S. H.; Senecal, K. J.; Kumar, J.; Samuelson, L. A. Electrospun Nanofibrous Membranes for Highly Sensitive Optical Sensors. Nano Lett. 2002, 2, 1273-1275.

(7) Yang, Y.; Wang, H.; Su, K.; Long, Y.; Peng, Z.; Li, N.; Liu, F. A Facile and Sensitive Fluorescent Sensor Using Electrospun Nanofibrous Film For Nitroaromatic Explosive Detection. J. Mater. Chem. 2011, 21, 11895-11900.

(8) Wang, X.; Lee, S. H.; Ku, B. C.; Samuelson, L. A.; Kumar, J. Synthesis and Electrospinning of A Novel Fluorescent Polymer PMMA-PM for Quenching Based Optical Sensing. J. Macromol. Sci., Part A: Pure Appl.Chem. 2002, A39, 1241-1249.

(9) Lan, A.; Li, K.; Wu, H.; Olson, D. H.; Emge, T. J.; Ki, W.; Hong, M.; Li, J. A Luminescent Microporous Metal-Organic Framework for the Fast and Reversible Detection of High Explosives. Angew. Chem., Int. Ed. 2009, 48, 2334-2338.

(10) Guo, L.; Zu, B.; Yang, Z.; Cao, H.; Zheng, X.; Dou, X. APTS and rGO Co-Functionalized Pyrenated Fluorescent Nanonets for Representative Vapor Phase Nitroaromatic Explosive Detection. Nanoscale 2014, 6, 1467-1473.

(11) Berliner, A.; Lee, M. G.; Zhang, Y.; Park, S. H.; Martino, R.; Rhodes, P. A.; Yi, G. R.; Lim, S. H. A Patterned Colorimetric Sensor Array for Rapid Detection of TNT at ppt Level. RSC Adv. 2014, 4, $10672-10675$.

(12) Hu, X.; Wei, T.; Wang, J.; Liu, Z. E.; Li, X.; Zhang, B.; Li, Z.; Li, L.; Yuan, Q. Near-Infrared-Light Mediated Ratiometric Luminescent Sensor for Multimode Visualized Assays of Explosives. Anal. Chem. 2014, 86, 10484-10491.

(13) Zhao, D.; Swager, T. M. Sensory Responses in Solution vs Solid State: A Fluorescence Quenching Study of Poly(iptycenebutadiynylene)s. Macromolecules 2005, 38, 9377-9384.

(14) Pablos, J. L.; Trigo-Lopez, M.; Serna, F.; Garcia, F. C.; Garcia, J. M. Solid Polymer Substrates and Smart Fibres for the Selective Visual Detection of TNT Both in Vapour and in Aqueous Media. RSC Adv. 2014, 4, 25562-25568.

(15) Senthamizhan, A.; Celebioglu, A.; Uyar, T. Flexible and Highly Stable Electrospun Nanofibrous Membrane Incorporating Gold Nanoclusters as an Efficient Probe for Visual Colorimetric Detection of $\mathrm{Hg}$ (II). J. Mater. Chem. A 2014, 2, 12717-12723.

(16) Anitha, S.; Brabu, B.; Rajesh, K. P.; Natarajan, T. S. Fabrication of UV Sensor Based on Electrospun Composite Fibers. Mater. Lett. 2013, 92, 417-420.

(17) Wu, X.; Li, H.; Xu, B.; Tong, H.; Wang, L. Solution-Dispersed Porous Hyperbranched Conjugated Polymer Nanoparticles for Fluorescent Sensing of TNT with Enhanced Sensitivity. Polym. Chem. 2014, 5, 4521-4525.

(18) Nie, H.; Lv, Y.; Yao, L.; Pan, Y.; Zhao, Y.; Li, P.; Sun, G.; Ma, Y.; Zhang, M. Fluorescence Detection of Trace TNT by Novel CrossLinking Electropolymerized Films Both in Vapor and Aqueous Medium. J. Hazard. Mater. 2014, 264, 474-480.

(19) Segui, F.; Qiu, X. P.; Winnik, F. M. An Efficient Synthesis of Telechelic Poly ( $\mathrm{N}$-isopropylacrylamides) and its Application to the Preparation of $\alpha, \omega$-Dicholesteryl and $\alpha, \omega$-Dipyrenyl Polymers. J. Polym. Sci., Part A: Polym. Chem. 2008, 46, 314-326.

(20) Danko, M.; Libiszowski, J.; Biela, T.; Wolszczak, M.; Duda, A. Molecular Dynamics of Star-Shaped Poly(L-lactide)s in Tetrahydrofuran as Solvent Monitored by Fluorescence Spectroscopy. J. Polym. Sci., Part A: Polym. Chem. 2005, 43, 4586-4599.

(21) Yuan, W.; Yuan, J.; Zhou, M.; Pan, C. Synthesis, Characterization, and Fluorescence of Pyrene-Containing Eight-Arm StarShaped Dendrimer-Like Copolymer With Pentaerythritol Core. J. Polym. Sci., Part A: Polym. Chem. 2008, 46, 2788-2798.

(22) Kalyanasundaram, K.; Thomas, J. K. Environmental Effects on Vibronic Band Intensities in Pyrene Monomer Fluorescence and Their Application in Studies of Micellar Systems. J. Am. Chem. Soc. 1977, 99, 2039-2044.

(23) Dong, D. C.; Winnik, M. A. The Py Scale of Solvent Polarities. Solvent Effects on the Vibronic Fine Structure of Pyrene Fluorescence and Empirical Correlations with ET and Y Values. Photochem. Photobiol. 1982, 35, 17-21.
(24) Dong, D. C.; Winnik, M. A. The Py Scale of Solvent Polarities. Can. J. Chem. 1984, 62, 2560-2565.

(25) Wang, Y.; La, A.; Brückner, C.; Lei, Y. FRET- and PET-Based Sensing in A Single Material: Expanding the Dynamic Range of an Ultra-Sensitive Nitroaromatic Explosives Assay. Chem. Commun. (Cambridge, U. K.) 2012, 48, 9903-9905.

(26) Ito, F.; Kakiuchi, T.; Sakano, T.; Nagamura, T. Fluorescence Properties of Pyrene Derivative Aggregates Formed Inpolymer Matrix Depending on Concentration. Phys. Chem. Chem. Phys. 2010, 12, 10923-10927.

(27) Deepak, V. D.; Asha, S. K. Photophysical Investigation into the Self-Organization in Pyrene-Based Urethane Methacrylate Comb Polymer. J. Phys. Chem. B 2009, 113, 11887-11897.

(28) Xiao, X.; Xu, W.; Zhang, D.; Xu, H.; Liu, L.; Zhu, D. Novel Redox-Fluorescence Switch Based on A Triad Containing Tetrathiafulvalene and Pyrene Units With Tunable Monomer And Excimer Emissions. New J. Chem. 2005, 29, 1291-1294.

(29) Ingratta, M.; Duhamel, J. Correlating Pyrene Excimer Formation with Polymer Chain Dynamics in Solution. Possibilities and Limitations. Macromolecules 2007, 40, 6647-6657.

(30) Winnik, F. M. Photophysics of Preassociated Pyrenes in Aqueous Polymer Solutions and in Other Organized Media. Chem. Rev. 1993, 93, 587-614.

(31) Winnik, M. A. End-to-End Cyclization of Polymer Chains. Acc. Chem. Res. 1985, 18, 73-79.

(32) Duhamel, J. Internal Dynamics of Dendritic Molecules Probed by Pyrene Excimer Formation. Polymers 2012, 4, 211-239.

(33) Duhamel, J. New Insights in the Study of Pyrene Excimer Fluorescence to Characterize Macromolecules and their Supramolecular Assemblies in Solution. Langmuir 2012, 28, 6527-6538.

(34) Kim, S. K.; Lee, D. H.; Hong, J. I.; Yoon, J. Chemosensors for Pyrophosphate. Acc. Chem. Res. 2009, 42, 23-31.

(35) Romero, T.; Caballero, A.; Tárraga, A.; Molina, P. A ClickGenerated Triazole Tethered Ferrocene-Pyrene Dyad for Dual-Mode Recognition of the Pyrophosphate Anion. Org. Lett. 2009, 11, 34663469.

(36) Martínez, R.; Zapata, F.; Caballero, A.; Espinosa, A.; Tárraga, A.; Molina, P. 2-Aza-1,3-butadiene Derivatives Featuring an Anthracene or Pyrene Unit: Highly Selective Colorimetric and Fluorescent Signaling of $\mathrm{Cu}^{2+}$ Cation. Org. Lett. 2006, 8, 3235-3238.

(37) Roy, B. C.; Chandra, B.; Hromas, D.; Mallik, S. Synthesis of New Pyrene-Containing, Metal-Chelating Lipids and Sensing of Cupric Ions. Org. Lett. 2002, 5, 11-14.

(38) Yamanaka, S. A.; Charych, D. H.; Loy, D. A.; Sasaki, D. Y. Solid Phase Immobilization of Optically Responsive Liposomes in Sol-Gel Materials for Chemical and Biological Sensing. Langmuir 1997, 13, 5049-5053.

(39) He, G.; Yan, N.; Yang, J.; Wang, H.; Ding, L.; Yin, S.; Fang, Y. Pyrene-Containing Conjugated Polymer-Based Fluorescent Films for Highly Sensitive and Selective Sensing of TNT in Aqueous Medium. Macromolecules 2011, 44, 4759-4766.

(40) Beyazkilic, P.; Yildirim, A.; Bayindir, M. Formation of Pyrene Excimers in Mesoporous Ormosil Thin Films for Visual Detection of Nitro-Explosives. ACS Appl. Mater. Interfaces 2014, 6, 4997-5004.

(41) Wang, Y.; La, A.; Ding, Y.; Liu, Y.; Lei, Y. Novel SignalAmplifying Fluorescent Nanofibers for Naked-Eye-Based Ultrasensitive Detection of Buried Explosives and Explosive Vapors. Adv. Funct. Mater. 2012, 22, 3547-3555.

(42) Hua, K. Y.; Deng, C. M.; He, C.; Shi, L. Q.; Zhu, D. F.; He, Q. G.; Cheng, J. G. Organic Semiconductors-Coated Polyacrylonitrile (PAN) Electrospun Nanofibrous Mats for Highly Sensitive Chemosensors via Evanescent-Wave Guiding Effect. Chin. Chem. Lett. 2013, 24, 643-646.

(43) Karagollu, O.; Gorur, M.; Gode, F.; Sennik, B.; Yilmaz, F. Synthesis, Characterization, and Ion Sensing Application of PyreneContaining Chemical Probes. Macromol. Chem. Phys. 2015, 216, 939949. 
(44) Sonar, P.; Soh, M. S.; Cheng, Y. H.; Henssler, J. T.; Sellinger, A. 1,3,6,8-Tetrasubstituted Pyrenes: Solution-Processable Materials for Application in Organic Electronics. Org. Lett. 2010, 12, 3292-3295.

(45) Salunke, J. K.; Sonar, P.; Wong, F. L.; Roy, V. A. L.; Lee, C. S.; Wadgaonkar, P. P. Pyrene Based Conjugated Materials: Synthesis, Characterization and Electroluminescent Properties. Phys. Chem. Chem. Phys. 2014, 16, 23320-23328.

(46) Zhao, Z.; Chen, S.; Lam, J. W. Y.; Wang, Z.; Lu, P.; Mahtab, F.; Sung, H. H. Y.; Williams, I. D.; Ma, Y.; Kwok, H. S.; Tang, B. Z. Pyrene-Substituted Ethenes: Aggregation-Enhanced Excimer Emission and Highly Efficient Electroluminescence. J. Mater. Chem. 2011, 21, $7210-7216$.

(47) Long, Y.; Chen, H.; Yang, Y.; Wang, H.; Yang, Y.; Li, N.; Li, K.; Pei, J.; Liu, F. Electrospun Nanofibrous Film Doped with a Conjugated Polymer for DNT Fluorescence Sensor. Macromolecules 2009, 42, 6501-6509.

(48) Novotney, J. L.; Dichtel, W. R. Conjugated Porous Polymers for TNT Vapor Detection. ACS Macro Lett. 2013, 2, 423-426.

(49) Tao, S.; Li, G.; Yin, J. Fluorescent Nanofibrous Membranes for Trace Detection of TNT Vapor. J. Mater. Chem. 2007, 17, 2730-2736.

(50) Senthamizhan, A.; Celebioglu, A.; Uyar, T. Ultrafast On-Site Selective Visual Detection of TNT at Sub-ppt Level Using Fluorescent Gold Cluster Incorporated Single Nanofiber. Chem. Commun. (Cambridge, U. K.) 2015, 51, 5590-5593.

(51) Senthamizhan, A.; Celebioglu, A.; Uyar, T. Real-Time Selective Visual Monitoring of $\mathrm{Hg}^{2+}$ Detection at ppt Level: An Approach to Lighting Electrospun Nanofibers Using Gold Nanoclusters. Sci. Rep. 2015, 5, 10403.

(52) Senthamizhan, A.; Uyar, T. In Electrospinning for High Performance Sensors; Macagnano, A., Zampetti, E., Kny, E., Eds.; Springer: Switzerland, 2015; Chapter 8, pp 179-204.

(53) Beyazkilic, P.; Yildirim, A.; Bayindir, M. Nanoconfinement of Pyrene in Mesostructured Silica Nanoparticles for Trace Detection of TNT in the Aqueous Phase. Nanoscale 2014, 6, 15203-15209.

(54) Niamnont, N.; Kimpitak, N.; Wongravee, K.; Rashatasakhon, P.; Baldridge, K. K.; Siegel, J. S.; Sukwattanasinitt, M. Tunable StarShaped Triphenylamine Fluorophores for Fluorescence Quenching Detection and Identification of Nitro-Aromatic Explosives. Chem. Commun. (Cambridge, U. K.) 2013, 49, 780-782.

(55) Stevens, J. S.; Newton, L. K.; Jaye, C.; Muryn, C. A.; Fischer, D. A.; Schroeder, S. L. M. Proton Transfer, Hydrogen Bonding, and Disorder: Nitrogen Near-Edge X-ray Absorption Fine Structure and Xray Photoelectron Spectroscopy of Bipyridine-Acid Salts and Cocrystals. Cryst. Growth Des. 2015, 15, 1776-1783.

(56) Stevens, J. S.; Byard, S. J.; Seaton, C. C.; Sadiq, G.; Davey, R. J.; Schroeder, S. L. M. Crystallography Aided by Atomic Core-Level Binding Energies: Proton Transfer versus Hydrogen Bonding in Organic Crystal Structures. Angew. Chem., Int. Ed. 2011, 50, 99169918. 